COMMUNICATION BRÈVE / BRIEF COMMUNICATION

\title{
Gestion de crise COVID en réanimation : afflux de patients et pénurie de médicaments
}

\author{
COVID surge in critical care: admission criteria and drug shortage
}

${\text { Bertrand } \text { Guidet }^{1 \star} \bullet \text { Georges France }}^{2}$

Reçu le 8 octobre 2020 ; accepté le 29 décembre 2020.

(c) SRLF 2021.

\section{Résumé}

La crise sanitaire liée à la COVID-19 a mis l'ensemble de l'hôpital en tension. Les services de réanimation ont été en première ligne pour prendre en charge les cas les plus graves. Le bilan des entrées et de l'occupation des lits de réanimation a été et est toujours un élément clé de mesure de l'intensité de la crise. La réanimation est une spécialité largement méconnue du grand public alors que des questions majeures se posent : priorité à la santé ou à l'économie ; perte de chance pour les malades non COVID du fait de la déprogrammation et accès à la réanimation pour les patients COVID.

L'augmentation de la demande de soins critiques a nécessité une extension des capacités d'hospitalisation par transformation de lits d'USC en réanimation, par création de néo-réanimation, voire dans certaines régions par réalisation en salle classique de soins habituellement réalisés en réanimation (DV vigile, CPAP, oxygène à haut débit humidifié). Des transferts inter-régionaux voire internationaux ont été nécessaires afin de soulager les régions en forte tension. Les facteurs limitants ont été multiples mais la principale difficulté a concerné la mobilisation de personnel formé à la réanimation et la relative pénurie de médicaments. L'inadéquation entre la demande et l'offre a conduit à édicter des règles de priorisation d'accès à la réanimation.

Cette revue aborde l'ensemble de ces questions et peut contribuer à une réflexion sur l'adaptation de l'hôpital pour faire face à une deuxième période épidémique.

Mots-clés : COVID, éthique, management, médicaments

\section{Abstract}

The health crisis linked to COVID-19 has put the whole hospital in tension. Intensive care units (ICU) have been on the front line to take care of the most serious cases. The number of new admission together with cumulative number of occupied intensive care beds have been and still are a key element in measuring the intensity of the crisis. Intensive care is a specialty largely unknown to the general public which is problematic when dealing with such difficult questions as should we give priority to health or the economy; is there a loss of chance for non-COVID patients?

The increase in the demand for critical care has necessitated an extension of hospitalization capacities by transforming intermediate care beds into ICU beds, by creating neo-ICU, or in some regions by carrying out care in regular wards that are usually performed in ICU (prone positioning, CPAP, humidified high flow oxygen). Inter-regional but also international transfer has been organized in order alleviating pressure for region with massive surge.

Among the several limiting factors, human resources with qualified personnel was a key element together the relative shortage of drugs. The mismatch between demand and supply has led to the proposal of rules for prioritizing access to ICU.

This review addresses all of these questions and can contribute to a reflection on the adaptation of the hospital to cope with a second epidemic period.

Keywords: COVID, ethic, management, drugs

\footnotetext{
${ }^{*}$ Pr Bertrand Guidet

Service Médecine Intensive Réanimation, Hôpital Saint-Antoine, Paris

$\nabla$ bertrand.guidet@aphp.fr

La liste complète des auteurs est disponible à la fin de l'article.
} 


\section{Introduction}

La crise sanitaire liée au SARS-Coronavirus-19 a mis les services de réanimation en première ligne. Le Directeur Général de la Santé (DGS) présentait tous les soirs l'état des lits occupés en réanimation puis un bilan des entrées et des sorties. Cet indicateur a été utilisé pour fixer la « couleur» des régions.

Or cette discipline était largement méconnue du grand public. Ceci est probablement lié au fait que peu de patients, relativement à d'autres spécialités, fréquentent les services de réanimation. Les médias présentaient souvent la réanimation à travers un prisme déformé insistant sur la gestion de la fin de vie (discussion de limitations et arrêt de traitement) ou d'acquisition d'infection (infection nosocomiale) et mélangeant la réanimation avec la médecine d'urgence ou l'anesthésie. Le message est tout autre : la réanimation est un lieu unique qui permet de prendre en charge les cas les plus graves avec une approche globale (médicale, technique et éthique).

La Direction Générale de l'Offre de Soins (DGOS) du Ministère de la santé mène depuis 2 ans une réflexion sur les soins critiques regroupant toutes les structures impliquées dans la prise en charge de patients instables ou potentiellement instables avec une proposition de structuration en deux niveaux pour la partie adulte : niveau 2 pour la Réanimation et niveau 1 pour les structures de soins intensifs de spécialité comme les unités de cardiologie (USIC) ou de neurovasculaire (USINV) et les USI polyvalentes qui remplaceront les USC. Toutes ces structures seront soumises à autorisation et devront donc répondre à un cahier des charges avec un focus sur la permanence médicale 24/24. Deux décrets devraient être publiés en 2021, l'un sur les structures et l'autre sur les conditions techniques de fonctionnement.

Afin de faire face à l'augmentation brutale des besoins en lits de réanimation, la réponse a été double avec d'une part une augmentation des lits de soins critiques et d'autre part une réflexion sur les critères d'admission. Un autre aspect est la relative pénurie de matériel et de médicaments qui interroge notre stratégie industrielle et notre indépendance internationale. La crise sanitaire a induit une double réflexion pour les réanimations.

\section{Extension des capacités de réanimation}

Cette question s'était déjà posée à l'occasion de la grippe aviaire en 2007 et de la grippe H1N1 en 2009. Plusieurs sociétés savantes s'étaient regroupées pour édicter des recommandations en insistant sur les facteurs limitant de l'extension de capacité [1]. Les difficultés ou points de blocage identifiés étaient : les locaux, les respirateurs, le monitorage (scope), les pousses seringues électriques, les coussins de positionnement, le matériel à usage unique, les médicaments, les matériels de protection (masque, surblouse) mais surtout le personnel. En situation de pandémie, de nombreux professionnels de santé ne peuvent pas travailler car ils sont eux-mêmes malades, ne peuvent faire garder leurs enfants ou ne peuvent se rendre à l'hôpital du fait de difficultés dans les transports. Ainsi, alors qu'il faut plus de monde au chevet des malades, il y a potentiellement moins de personnel disponible. De plus, il faut que le personnel ait une compétence en soins critiques. II faut noter que dans plusieurs pays, il existe une surspécialisation pour les infirmières de soins critiques (Canada, Suisse...). II faut donc en France, une reconnaissance spécifique du métier d'IDE de réanimation, une formation adaptée et une rémunération attractive. II faut par ailleurs des médecins réanimateurs formés en nombre suffisant avec un exercice exclusif de la réanimation, garant de la qualité de l'équipe et de l'expertise individuelle.

Dans le cadre de la COVID, nous avons rapidement été alertés (Italie du Nord et Grand Est de la France) que nous devrions étendre nos capacités et créer des néoréanimations. En quelques jours, grâce à la mobilisation de tous les corps de métier de l'hôpital, les capacités de réanimation ont été considérablement augmentées. En Île-de-France, le nombre de lits de réanimation est passé de 1100 à 2700 au pic de l'épidémie en avril 2020 avec seulement 250 lits pour les patients non-Covid. Cette répartition a été rendue possible par la déprogrammation ainsi que par le confinement conduisant à moins d'hospitalisations pour un accident traumatique. En revanche, la réduction du capacitaire non-Covid a pu s'accompagner de perte de chance comme ce qui a pu être montré pour le cancer [2]. La reproduction de ce modèle est beaucoup plus aléatoire pour l'hiver du fait de plusieurs facteurs : période de l'année avec une saturation des lits particulièrement dans les secteurs de soins critiques, déprogrammation seulement partielle, absence d'entraide inter-régionale du fait de la circulation du virus dans pratiquement toutes les régions de France, fatigue du personnel.

II faut noter que l'augmentation excessive du nombre de lits de réanimation s'accompagne inéluctablement d'une réduction de la qualité des soins [3].

La projection d'un relatif déficit de lits de réanimation pose la question des règles de priorisation pour l'accès à ces secteurs spécialisés en tension.

\section{Priorisation pour l'admission en réanimation}

\section{Le contexte}

Les règles d'attribution des lits et donc l'ordre de priorité dépendent de la situation de tension. En situation normale, le principe égalitaire doit s'appliquer; les srlf 
personnes pouvant bénéficier des soins de réanimation doivent pouvoir être admises sans considération d'âge, de conditions sociales, de religion... Ce principe éthique a été rappelé par le Comité Consultatif National d'Ethique (CCNE) le 17 Novembre [4] mais il est mis à mal lorsque les capacités d'admission sont dépassées. Dans la situation caricaturale où il n'existerait plus qu'un seul lit libre de réanimation, l'application stricte du principe égalitaire conduirait à un choix par tirage au sort ou selon le principe du premier présent, premier admis [5]. L'intégration de facteurs de choix s'apparente à une approche utilitaire qui est dénoncée par certains.

Ainsi, on peut schématiquement identifier trois situations : la bonne où les pratiques ne sont pas remises en question mais où il existe déjà une sélection comme ce qui a été montré pour les personnes âgées [6] ; la mauvaise où les capacités d'admission ont été augmentées (voir supra) et l'affreuse où les capacités de réanimation sont dépassées et qui demande des choix ne relevant plus de la médecine mais de la société [7].

La question fondamentale est l'évaluation du rapport bénéfice-risque. Notre obsession est d'éviter à tout prix les pertes de chances. En miroir, il ne faut pas admettre en réanimation un malade qui ne va pas bénéficier des traitements lourds, douloureux pour le patient et sa famille (obstination déraisonnable).

\section{Les grands principes de priorisation en période} de crise sanitaire

Les conditions des décisions de fin de vie ont été précisées par la loi Clayes-Leonetti. La décision de ne pas admettre un patient en réanimation peut s'apparenter à une décision de limitation des traitements. II faut rappeler les grands principes éthiques en insistant sur les spécificités de la réanimation. Des recommandations ont été élaborées par des médecins issus des spécialités MIR, MAR et MU et ont été validées par l'Agence Régionale de Santé d'Île-de-France (ARSIF) [8]. Une version en langue anglaise est disponible [9].

La décision reste de la responsabilité d'un seul médecin, mais elle est prise en concertation avec l'équipe soignante. C'est le principe de la collégialité. Les volontés et valeurs du patient doivent être respectées : c'est le principe d'autonomie.

L'état antérieur du patient conditionne son risque de décès et sa capacité à faire face à l'agression que constitue un séjour lourd de réanimation. Parmi les multiples facteurs proposés, l'âge a été utilisé comme facteur de "sélection ». Cette approche fondée sur le seul critère de l'âge est jugée discriminatoire car pouvant s'apparenter à du racisme " anti-vieux » (âgisme). D'ailleurs, la société américaine de gériatrie a affiché son opposition formelle à l'utilisation du critère de l'âge comme facteur devant être considéré dans le tri à l'admission en réanimation en période de crise COVID [10,11].

De nombreuses recommandations émises par les pays Européens intègrent l'âge comme un des facteurs à considérer mais sans fixer de valeur seuil [12] notamment en Angleterre [13] et en Suisse [14]. Les collègues Italiens ont proposé un seuil à 70 ans [15]. Cette attitude repose sur plusieurs arguments.

La démonstration du bénéfice de la réanimation pour les personnes âgées reste à faire. En dehors de la crise COVID, dans une étude prospective, randomisée, en cluster, nous n'avons pas montré de bénéfice d'une stratégie d'admission systématique versus une stratégie basée sur les pratiques habituelles. Le critère de jugement était la mortalité à 6 mois mais également des critères qualitatifs (perte d'autonomie et qualité de vie perçue) [16]. Cependant, dans une autre étude multicentrique européenne, nous avons montré que le poids de la fragilité évaluée par l'échelle Clinical Frailty Scale (CFS) était plus important que l'âge pour prédire la mortalité [17]. Ainsi, même si l'âge est un facteur de risque indépendant de mortalité (encore plus dans le COVID), l'âge seul ne doit pas être utilisé pour « sélectionner » les malades devant être admis en réanimation. L'ajout d'autres facteurs d'évaluation gériatriques en plus du CFS n'ajoute rien à la prédiction [18]. Nous avons proposé de systématiser le recueil du CSF pour mieux caractériser une personne âgée comme ce qui est fait en Grande-Bretagne [13].

Les patients non admis en réanimation car considérés comme trop graves et/ou ne pouvant bénéficier de la réanimation doivent être pris en charge dans des structures adaptées. Ceci suppose la mise en place d'une cellule d'aide à la décision d'orientation avec traçabilité des décisions et une structure d'hospitalisation de soins palliatifs. II est en effet fondamental d'assurer le confort du patient avec prise en charge de la douleur, de l'anxiété, de l'agitation, de la dyspnée, de l'encombrement bronchique, et de l'isolement social. Ces soins doivent être respectueux de la personne et de sa dignité.

\section{Le processus décisionnel}

Il comporte plusieurs étapes avec une anticipation d'un éventuel recours aux soins critiques dès l'évaluation clinique initiale ; un recueil des éléments pertinents à l'analyse clinique de la situation et en cas d'admission en réanimation, une réévaluation périodique prenant en compte la réponse aux thérapeutiques administrées sur les défaillances d'organe avec éventuelle décision d'introduire des limitations ou un arrêt de traitement (figure 1) [19].

Dans tous les cas, une communication claire, loyale et sincère doit être assurée avec l'entourage et les proches, 


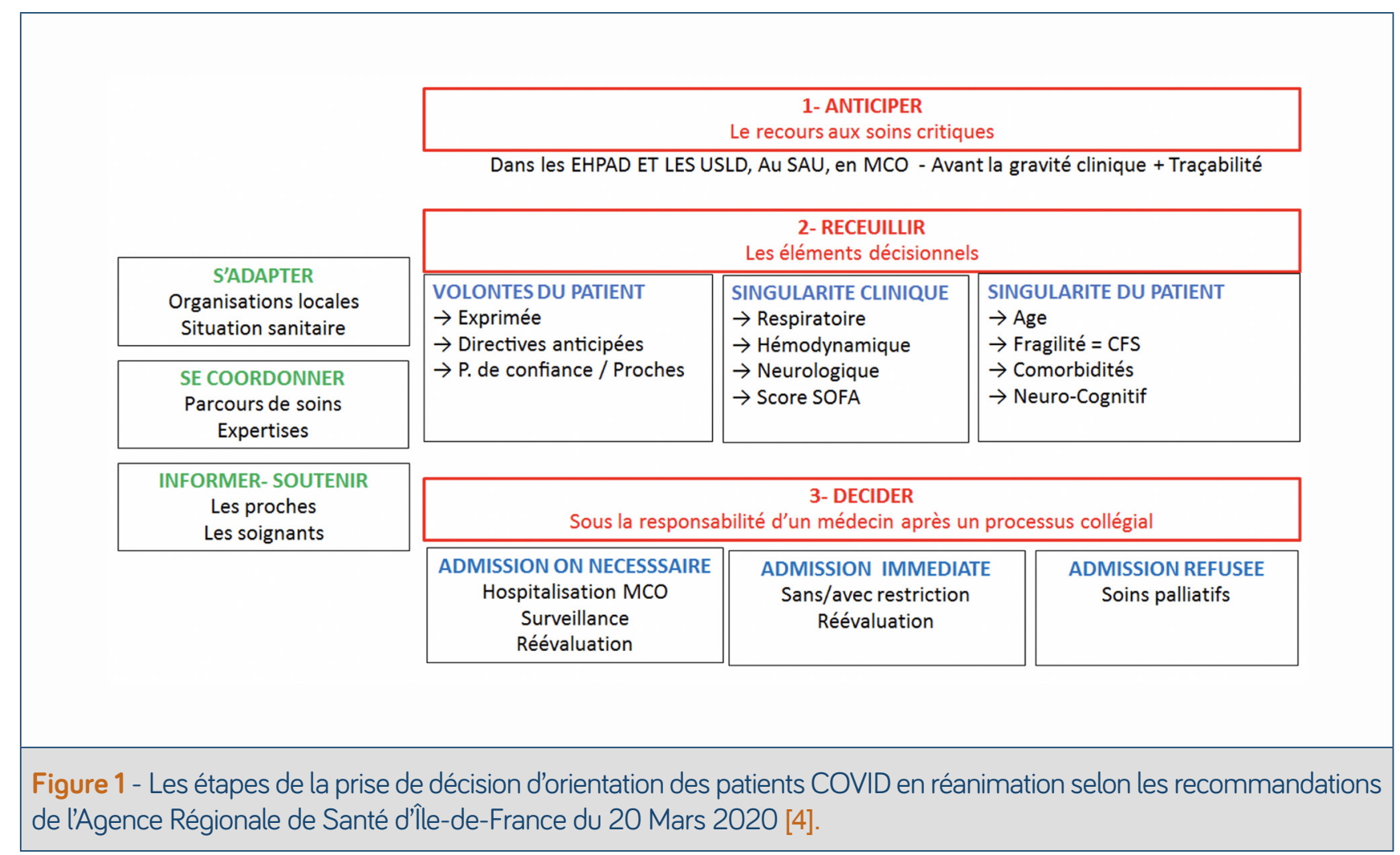

première étape de leur accompagnement. La prise en charge des détresses familiales dans cette période de restriction des visites est fondamentale avec des entretiens réguliers, aide de psychologues et psychiatres.

Le débat sur l'accès aux services de réanimation en situation de crise sanitaire fait rage en Europe et aux USA avec des mises en accusation des médecins mais aussi des gouvernements par des groupes de personnes se jugeant stigmatisés et défavorisés avec un focus sur les patients âgés. Un compte rendu biaisé et partisan de l'entretien donné au journal Le Monde par E. Ciotti, rapporteur de la commission d'enquête du parlement, a appelé de notre part une réponse dans Le Monde [20]. Une réflexion européenne est conduite sous l'égide de la fondation Konrad Adenauer. La question de l'accès à la réanimation en situation de crise sanitaire doit être abordée avec une dimension sociétale et non laissée à la seule appréciation des praticiens de terrain [21].

\section{Les matériels et médicaments}

L'état des stocks de matériel et de médicaments indispensables à la prise en charge des patients est suivi au niveau national, régional et local. Parmi les points de blocage, il y avait le matériel à usage unique mais aussi du matériel comme les PSE, les coussins de positionnement, les surblouses, les respirateurs, et bien sûr les masques
FFP2 et chirurgicaux). La liste des lignes particulièrement regardées est indiquée sur le tableau 1. Nous avons choisi de réaliser un focus sur les médicaments.

Médicaments, leur principe actif et leur matières premières [21-25]

La pandémie de Covid-19 a mis en évidence la difficulté pour la France à maîtriser ses approvisionnements en médicaments ce qui interroge sur notre perte de souveraineté vis-à-vis du médicament. Des tensions sont apparues sur certaines classes dont les curares, le Midazolam avec des conséquences sur les protocoles de prise en charge.

La réflexion de relocalisation doit intégrer la complexité de l'environnement du médicament: chaîne d'approvisionnement (ou Supply Chain) globalisée et complexe dont on ne voit souvent que le dessus de l'iceberg, associée à une réglementation internationale fragmentée et disparate [21]. Au-delà de la France et de l'UE, l'accès aux médicaments doit être réfléchi de façon globale [22]. C'est une réalité pour toutes les grandes multinationales, mais cela l'est aussi pour les entreprises régionales ou nationales : la fabrication finale des médicaments et surtout celle de leur principe actif sont réalisées dans des pays tiers et hors du contrôle de la France de I'UE. srlf 
Tableau 1 - Suivi de stock de matériel et de médicaments

\begin{tabular}{|c|c|}
\hline Matériel & Médicaments \\
\hline Masques & Atracurium \\
Lunettes & Cisatracurum \\
Surblouses & Etomidate \\
Kimonos & Midazolam \\
Ponchos & Propofol \\
Combinaisons & Dexamethasone \\
Tabliers & Ketamine \\
Pyjamas & Norepinephrine \\
Charlottes & Remifentanil \\
Visières & Sufentanil \\
Gants d'examens & Tocilizumab \\
Pansements anti-escarre & \\
Ecouvillons & \\
Filtres patient & \\
Filtres machine & \\
Masques 02 & \\
haute concentration & \\
Système clos & \\
d'aspiration endotrachéale & \\
SHA & \\
Casaques stériles & \\
Cants stériles & \\
\hline
\end{tabular}

À ce stade, il est fondamental de différencier les médicaments innovants des médicaments génériquables. Dans la plupart des cas pour les médicaments innovants, la fabrication reste dans la région (Europe, États-Unis...) du laboratoire à l'origine de la molécule et ceci pour une raison économique (les marges restent élevées), mais aussi pour protéger le savoir-faire et la propriété industrielle.

La situation est bien différente pour les médicaments tombés dans le domaine public et génériquables. Pour ceux-là et leurs génériques, les marges sont bien inférieures et l'optimisation du coût est un enjeu majeur. Même si un pourcentage raisonnable de la fabrication finale (formulation et conditionnement) est encore réalisé en Europe (environ $60 \%$-source European Federation of Pharmaceutical Industries and associations), la plupart des laboratoires se fournissent en matières premières sur le marché mondial. Les principes actifs et leurs matières premières, comme beaucoup des excipients, sont fabriqués en majeure partie hors de nos frontières et des frontières de l'UE. La Chine et l'Inde en sont les principaux fournisseurs et ceci depuis plusieurs décennies (le basculement a eu lieu entre 1990 et 2000).

\section{Comment relocaliser?}

D'évidence, le premier point à reconsidérer est l'aspect économique [24] :

- Réajuster les médicaments anciens à un prix permettant de couvrir cette relocalisation et cibler « les médicaments anciens indispensables ॥

- Considérer pour ces médicaments indispensables (génériquables et leurs génériques) un prix de remboursement garantissant une source d'approvisionnement sûr et pérenne

- Associer une politique du médicament favorisant/ subventionnant les investissements dans ce secteur et coordonner ces actions au niveau Européen.

Enfin, sur un aspect technico-règlementaire, il est indispensable d'avoir une meilleure transparence et une meilleure visibilité de la Supply Chain en partant des fournisseurs de la matière première aux médicaments délivrés. Même si une relocalisation n'est pas envisageable, de nombreuses mesures réglementaires peuvent être considérées pour harmoniser les contraintes réglementaires et faciliter leurs disponibilités. En cette année de mobilisation mondiale face à la pandémie du COVID-19, comme pour la COP21 pour le climat, la France pourrait lancer une initiative pour la santé avec une Conférence Internationale du Médicament et en prendre le leadership au niveau européen [25].

\section{Conclusion}

La réanimation, discipline relativement jeune, a été découverte par beaucoup à l'occasion de la pandémie de COVID. II s'agit d'une spécialité transversale avec des zones d'interface multiples (ville avec la médecine d'urgence préhospitalière), services cliniques de l'hôpital, services médicotechniques.

La Réanimation couvre pratiquement tous les champs de la médecine, mobilise une expertise clinique polyvalente, une habileté technique et une réflexion éthique comme illustré par le débat autour de l'accès à ces structures mises en tension par la crise sanitaire. Les capacités de communication avec l'ensemble des intervenants de l'hôpital et la collaboration avec l'équipe soignante sont garantes de la qualité des soins. La disponibilité du matériel et des médicaments est un point clés mais qui ne va pas sans un personnel qualifié en nombre suffisant qu'on saura garder afin d'éviter cette fuite de compétence. 


\section{Éthique et consentement à la participation}

Revue générale sans besoin de consentement à la participation.

\section{Conflits d'intérêt}

Les auteurs déclarent ne pas avoir de conflit d'intérêts.

\section{Sources de financement}

Les études ICE-CUB ont été financées par le PHRC. Les études VIP ont été endossées par l'ESICM.

\section{Remerciements}

À tous les collègues qui ont inclus les patients dans les études ICECUB et VIP.

Toutes ces études ont bénéficié du soutien de la DRCl et de l'URC-Est. À tout le personnel du service MIR de l'Hôpital Saint-Antoine pour leur dévouement dans la prise en charge des malades pendant la première vague COVID.

\section{Affiliations}

${ }^{1}$ Sorbonne Université, INSERM

Institut Pierre Louis d'Epidémiologie et de Santé Publique

AP-HP, Hôpital Saint-Antoine

Service de Médecine Intensive Réanimation

F75012, Paris, France

${ }^{2}$ Quality Expert. Consultant. Vice-President Global Quality (Retired) GSK

Membre de l'Académie Nationale de Pharmacie

Expert groupe Qualité ICH (International Conference of Harmonisation) Avenue Reverdil 14, 1260 Nyon, Switzerland

\section{Références}

1. Guery B, Guidet B, Beloucif S, et al (2007) Organization of intensive care units, in case of pandemic avian flu. Med Mal Infect. 37: S194203. DOI : 10.1016/j.medmal2007.09.005

2. Hanna TP, King WD, Thibodeau S et al (2020) Mortality due to cancer treatment delay: systematic review and meta-analysis. 371: $\mathrm{m} 4087$. DOI : 10.1136/bmj.m4087

3. Depuydt P, Guidet B (2020) Triage policy of severe covid-19 patients: what to do now? Ann Intensive Care

4. Comité Consultatif National d'Éthique (16/11/2020) Enjeux éthiques de la prise en charge et de l'accès aux soins pour tous en situation de forte tension liée à l'épidémie de Covid-19. www.ccne-ethique.fr Consulté le 15/01/2021. Document en ligne: https: //www.ccne-ethique. $\mathrm{fr} /$ sites/default/files/ccne___saisine_triage.pdf

5. Guidet B (07/11/2020) Des malades ne seront pas pris en réanimation. On s'y prépare. Le Monde. www.lemonde.fr Consulté le 15/01/2021. Article de presse en ligne : https: //www.lemonde.fr/ planete/article/2020/11/07/covid-19-des-malades-ne-seront-pas-pris-enreanimation-on-s-y-prepare_6058859_3244.html

6. Boumendil A, Angus DC, Guitonneau AL, et al (2012) Variability of intensive care admission decisions for the very elderly. Plos One 7: e34387. DOI : 10.1371/journalpone.0034387

7. Flaatten H, Beil M, Guidet B et al (2020) The good, the bad and the ugly: pandemic priority decisions and triage. J Med Ethics. medethics-2020-106489. DOI : 10.1136/medethics-2020-106489
8. Agence Régionale de Santé (ARS) - île-de-France (20/03/2020) COVID-19: Décision d'admission des patients en réanimation et soins critiques - V1 - 20/03/2020. www.ledefrance.ars.sante.fr PDF consulté le 15/01/2021. PDF en ligne: https: //www.ledefrance.ars.sante.fr/system/ files/2020-03/010_ARSIdF-CRAPS_2020-03-19_Doctrine_Criteresadmission_Soins-Critiques.pdf

9. Azoulay E, Beloucif S, Guidet B, et al (2020) Admission Decisions to Intensive Care Units in the Context of the Major Covid-19 Outbreak: local guidance from the COVID-19 Paris-Region Area. Crit Care 24: 293. DOI : 10.1186/s13054-020-03021-2

10. Farrell TW, Ferrante LE, Brown T et al (2020) AGS Position Statement: Resource Allocation Strategies and Age-Related Considerations in the COVID-19 Era and Beyond. J Am Geriatr Soc. 68: 1136-1142. DOI : 10.1111/jgs.16537

11. Farrell TW, Francis L, Brown T, et al (2020) Rationing Limited Healthcare Resources in the COVID-19 Era and Beyond: Ethical Considerations Regarding Older Adults. J Am Geriatr Soc. 68: 1143-1149. DOI : 10.1111/ jgs.16539

12. Joebges S, Biller-Andorno N (2020) Ethics guidelines on COVID-19 triage-an emerging international consensus. Crit Care 24: 201. DOI : 10.1186/s13054-020-02927-1

13. National Institute for Health and Care Excellence. Acute and critical care. Consulté le 15/01/2021. En ligne : https: //www.nice.org.uk/ guidance/health-and-social-care-delivery/acute-and-critical-care

14. Académie Suisse des Sciences Médicales (2020) Pandémie Covid-19: triage des traitements de soins intensifs en cas de pénurie des ressources. www.samw.ch Consulté le 15/01/2021. Téléchargeable en ligne: https: //www.samw.ch/fr/Ethique/Apercu-des-themes/Medecineintensive.html

15. Riccioni L, Bertolini G, Giannini A et al (Gruppo Di Lavoro Siaarti - Società Italiana Di Anestesia Analgesia Rianimazione E Terapia Intensiva) (2020) Raccomandazioni di etica clinica per l'ammissione a trattamenti intensivi e per la loro sospensione, in condizioni eccezionali di squilibrio tra necessità e risorse disponibili. Recenti Prog Med. 111: 207-211. http: //dx.doi.org/10.1701/3347.33183

16. Guidet B, Leblanc G, Simon T, et al (2017). Effect of systematic intensive care unit triage on long-term mortality among critically ill elderly patients in France: a randomized clinical trial JAMA 318: 1450-9. DOI : 10.1001/jama.2017.13889

17. Flaatten $\mathrm{H}$, De Lange D, Morandi A, et al (2017) The impact of frailty on ICU and 30-day mortality and the level of care in very elderly patients ( 280 years). Intensive Care Med 43: 1820-1828. DOI : 10.1007/ s00134-017-4940-8

18. Guidet B, Dylan W de Lange, Boumendil A, et al (2020) The contribution of frailty, cognition, activity of daily life and comorbidities on outcome in acutely admitted patients over 80 years in European ICUs: The VIP2 study. Intensive Care Med 46: 57-69. DOI : 10.1007/ s00134-019-05853-1

19. Guidet B, Flaatten H, Boumendil A, et al (2018) Withholding or withdrawing of life sustaining therapy in very elderly patients $(\geq 80$ years) admitted to the Intensive Care Unit. Intensive Care Med 44: 1027-1038. DOI : 10.1007/s00134-018-5196-7

20. Guidet B, Pautas E, Riou B et al (3/08/2020) « Non, les hôpitaux franciliens n'ont pas trié les patients Covid selon leur seul âge pour srlf 
l'accès à la réanimation! ». Le Monde, www.lemonde.fr Article consulté le 15/01/2021. En ligne: https: //www.lemonde.fr/idees/article/2020/08/03/ non-les-hopitaux-franciliens-n-ont-pas-trie-les-patients-covid-selon-leurseul-age-pour-l-acces-a-la-reanimation_6048052_3232.html

21. Gelinsky K (02/09/2020) A qui ira le dernier ventilateur? Un dialogue franco-allemand sur l'allocation des ressources en réanimation à l'ère de la COVID-19. Entretien avec le Professeur Christian Jung et le Professeur Bertrand Guidet. Konrad Adenauer Foundation, https: //www.kas.de/. Consulté le 15/01/2021. En ligne : https: //www.kas. de/documents/252038/7995358/A+qui+ira+le+dernier+ventilateur.pdf/ fd550650-3bb6-4727-deca-60e53811a423?version=1.1\&t=1601305457111

22. Académie nationale de Pharmacie (20/06/2018) «Indisponibilité des médicaments » www.acadpharm.org Consulté le 15/01/2021. En ligne : https: //www.acadpharm.org/dos_public/2018_06_20_AnP_ RAPPORT_INDISPONIBILITE_MED_VF1.pdf

23. Sénat (27/09/18) « Pénuries de médicaments et de vaccins: renforcer l'éthique de santé publique dans la chaine du médicament ». Consulté le 15/01/2021. En ligne : https: //www.senat.fr/rap/r17-737/r17-7371.pdf

24. Biot J, rapport public pour le Ministère des Solidarités et de la Santé $(04 / 02 / 2020)$ « Mission stratégique visant à réduire les pénuries de médicaments essentiels : rapport au premier ministre ». Consulté le 15/01/2021. En ligne : https: //www.vie-publique.fr/rapport/274702-

mission-strategique-pour-reduire-les-penuries-de-medicaments-essentiels

25. Parlement européen (17/09/2020) «Pénurie de médicamentscomment faire face à un problème émergent ». Consulté le 15/01/2021. En ligne: https: //www.europarl.europa.eu/doceo/document/TA-9-20200228_FR.html 
